Vietnam Journal of Mechanics, VAST, Vol.34, No. 4 (2012), pp. $237-245$

\title{
LOW VOLTAGE OPERATION ELECTROSTATIC COMB DRIVE MICRO-MIRROR FOR LASER SCANNING DISPLAY
}

\author{
Hoang Manh Chu ${ }^{1}$, Hung Vu Ngoc ${ }^{1}$, Kazuhiro Hane ${ }^{2}$ \\ ${ }^{1}$ Hanoi University of Science and Technology, Vietnam \\ ${ }^{2}$ Tohoku University, Japan
}

\begin{abstract}
Electrostatic comb drive micro-mirror has limitation of high operation voltage, which has limited its practice use. Recent developments in decreasing operation voltage are promising for mobile micro-mirror. Especially, the operation voltage is decreased substantially by removing the air friction of comb-drive micro-mirror at a high resonant frequency when the mirror is operated at low pressure. Removing simultaneously the air friction and anchor loss, electrostatic comb-drive micro-mirror operating at a few voltages at a high resonant frequency becomes possible. This paper presents our recent developments as well as emerging techniques introduced in literature for decreasing the operation voltage of electrostatic comb drive micro-mirror. Methods for decreasing the operation voltage at large rotation angle and high scan frequency for application in the laser scanning display are focused to discuss in this paper.
\end{abstract}

Keyword: Micro-mirror, vertical electrostatic comb drive, air damping, Q factor.

\section{INTRODUCTION}

Micro-mirror is known as a most successfully developed MEMS device for promising applications in laser medical imaging, optical communication and micro-display. Compared to actuation mechanisms such as magnetic and piezoelectric, the electrostatic mechanism has advantages of major fabrication technology, mass production with high reliability and low power consumption. However, the limitation of the micro-mirror driven electrostatically is the high operation voltage $(\sim 100 \mathrm{~V})$ required for a large rotation angle at the high scan frequency $(\sim 15 \mathrm{kHz})$. This is due to the large stiffness of torsion hinge for the high scan frequency. The requirements for laser scanning display are a large mirror-size scan-angle product and a high scan frequency. The mirror-size scan-angle product and scan frequency for the low resolution (QVGA) are 3.9 deg. $\mathrm{mm}$ and $8 \mathrm{kHz}$, respectively. Those for the high resolution (HTDV) are $23.3 \mathrm{deg} . \mathrm{mm}$ and $36 \mathrm{kHz}$, respectively [1]. Decreasing the operation voltage of electrostatically driven micro-mirror can be carried out using soft spring such as thin-film torsion bar [2] or SU8 polymer torsion bar [3]. However, the mentioned methods lead to decreasing scan frequency, which is not suitable for the requirement of high resolution scan display. In other effort, researchers proposed methods for decreasing the operation voltage while keeping the high scan frequency for the large 
angle scan micro-mirror. To decrease the driving voltage and increase the scan angle, the optimizations of vertically self-assembled combs is introduced $[4,5]$. The comb actuator with narrow gap was also effective for increasing the electrostatic force [6]. Specially, it was recently reported that the operation voltage was decreased substantially by decreasing the air friction of comb-drive micro-mirror at a high resonant frequency when the mirror was operated at low pressure [7], [8]. In order to use this advantage for mobile micro-mirror, the vacuum packaging techniques for the micro-mirror are also introduced using anodic bonding [9] and TO8 metal can [10].

As a quick reference for the research and development of the mobile micro-mirror, we review our recent developments as well as emerging techniques introduced in literature for decreasing the operation voltage of the vertical electrostatic comb-drive micro-mirror. The comb actuator with narrow gap and out-of-plane comb drive arrangement such as vertically self-assembled combs, dual comb, bidirectional comb drive and angle vertical comb drive are reviewed. The research results by the authors from in-plane comb drive arrangement, energy loss due to air friction and anchor for decreasing the operation voltage of the vertical electrostatic comb-drive micro-mirror are presented.

\section{ELECTROSTATIC COMB DRIVE ACTUACTOR}

Electrostatic actuation utilizes coulombic attraction between two bodies to induce displacement. The electrostatic actuator is preferred since the device can be completed within a chip, making integration easy. The actuator consumes little power. Heat dissipation is zero, in principle, as there are no steady-state currents caused by capacitive loads. The degradation in the device reliability due to heat generation can be prevented.

Micro-mirrors operate based on the electrostatic actuation, which is common constructed on two kinds of actuators: one is the parallel plate-type electrostatic actuator $[11,12]$; the other is the vertical comb-drive electrostatic actuator [2-5, 7-10]. Compar-
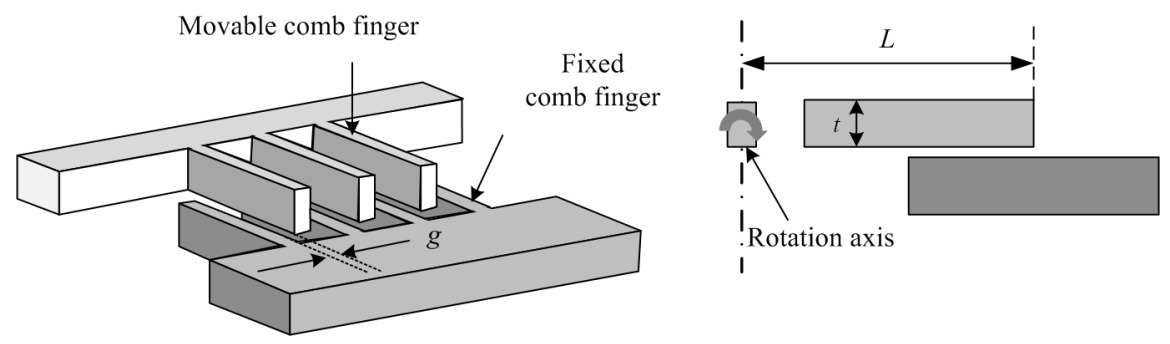

Fig. 1. Schematic drawing of a vertical comb-drive electrostatic actuator

ing with the parallel plate-type actuator, the vertical comb-drive actuator is interested and studied widely for application. This is due to the vertical comb-drive actuator having the advantages of high electrostatic force and large continuous rotation angle without limited by pull-in effect such as the parallel plate-type electrostatic actuator. The comb drive consists of two sets of capacitor banks, each containing a number of capacitors $N$ in parallel. $F_{e}$ is evaluated as follows, $F_{e}=A N V^{2} / g$, where $A$ is a constant depending 
on the geometrical dimensions of comb fingers, $N$ is the number of comb finger pairs, $V$ is the driving voltage, and $g$ is the gap between fixed and movable comb fingers (see Fig. 1).

\section{METHODS FOR DECREASING OPRATION VOLTAGE OF VERTICAL ELECTROSTATIC COMB DRIVE MICRO-MIRROR}

As mentioned above, the vertical electrostatic comb-drive actuator is superior to the parallel plate-type electrostatic actuator. Therefore, micro-mirrors driven by the vertical comb-drive electrostatic actuation are interested and studied for application. Methods for decreasing the operation voltage of vertical electrostatic comb drive micro-mirror are focused to discuss here.

\subsection{Comb drive with narrow gap}

The electrostatic force of comb drive is proportional directly to the square of applied voltage and inversely to the gap between fixed and movable comb fingers. Therefore, the operation voltage micro-mirror can be decreased by reducing the gap between fixed and movable comb fingers. However, when the gap is decreased, the instability of comb drive increases [6]. Moreover, decreasing the gap is not an easy process because the gap between comb fingers depends on the resolution of lithography and the effect of edge ratio in deep etching process [13].

\subsection{Design of comb electrode structure}

For the mirror with the conventional rectangular comb-drive, the micro-mirror is possible to operate at low voltage by means of either reducing the gap between comb fingers as mentioned above or increasing the number of combs in electrostatic actuator. In the second case, the size of the micro-mirror will increase, which is not desirable for miniature [14]. Following analyses are for methods such as adding fixed comb fingers and comb drive reconfiguration. For convenience, we relatively classify into two groups: (1) out-of-plane comb drive arrangement and (2) in-plane comb drive arrangement.

\subsubsection{Out-of-plane comb drive arrangement}

The driving electrodes of conventional vertical comb drive mirror are demonstrated by a cross-section view as in Fig. 2 (a). Two sets of fixed comb fingers with two potentials V1 and V2 are electronically separated. Two sets of movable comb fingers on both opposite sides are normally grounded. The operation of the mirror is based on electrostatic force imbalance by contacting the two sets of fixed comb electrodes with a potential different from that of the moving comb electrodes. The electrostatic force imbalance can be generated by asymmetries due to fabrication and/or by choosing different potentials for the two sets of fixed comb fingers. However, at the same time the only electrostatic force each side is available for driving the mirror. In order to increase the driving force but not decrease resonant frequency, in addition to lower side fixed comb electrodes as in Fig. 2 (a), fixed comb electrodes with electronically isolated potentials V3 and V4 are added on the upper of the moving comb fingers as shown in Fig.2 (b) [5]. This dual vertical comb structure also has an advantage of decreasing vertical movement of the mirror induced by the unbalanced vertical force with respect to the tensional axis as compared with that of 
the conventional comb electrode structure (Fig. 2 (a)). However, this method needs the complicated assemble process, which leads to misalignment between the fixed and moving comb fingers.

Using the multilevel beam SOI-MEMS process [15], Milanovic et al. demonstrated a micro-mirror with a down comb drive set and an up comb drive set, each on opposing sides of the rotation axis as shown in Fig. 2(c). The electrostatic driving force on both sides at the same time (Fig. 2 (c)) is possible to carry out similar to that of the comb electrodes arrangement in Fig. 2 (b). The same as the comb drive structure in Fig. 2 (b), the pure rotation motion is obtained by applying equivalent actuation voltage to comb electrodes V1 and V2, and grounding movable comb electrodes.

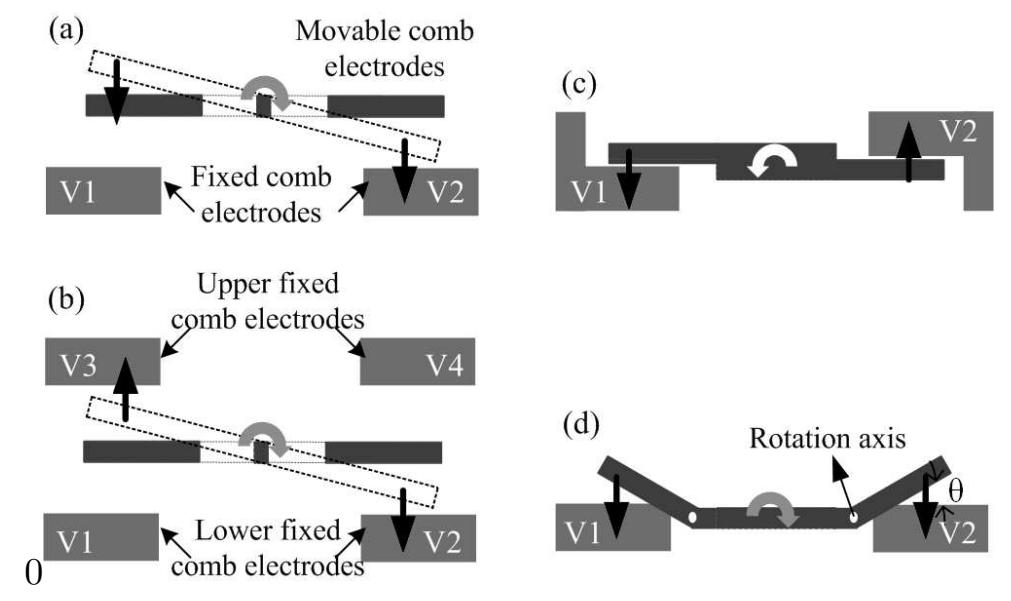

Fig. 2. Structure of comb drive: (a) conventional comb drive, (b) Structure of dual comb electrode, (c) bidirectional comb drive, and (d) angle comb drive

In another development, the micro-mirror with the angle vertical comb drive using reflow of photoresist is reported as shown in Fig. 2 (d) [16]. The moving fingers are patterned in the same layer as the fixed fingers and then tilted upward using the surface tension of the photoresist. The fingers are self-aligned. Compared to staggered vertical comb, the angle vertical comb drive can generate a large overlap capacitance between the moving and fixed comb fingers. The maximum scan angle of the angle vertical comb drive increases up to $60 \%$ compared to that of the staggered vertical comb (Fig. 2 (a)) for the same comb-finger dimensions with the enlarged stable region. To form the angular vertical combs, the method based on the plastic deformation of silicon was also suggested [17]. Compared with the method using reflow of photoresist above, the plastic deformation of silicon allows forming more robust, higher scanning frequency device although the fabrication process is performed at high temperature $\left(900^{\circ} \mathrm{C}\right)$ to form the permanent plastic deformation of silicon torsion spring. An electrostatic torsional micro-mirror driven independently in two orthogonal directions using two sets of self-aligned angular vertical combs are also reported [18]. The self-aligned angular vertical combs are fabricated based on a plastic deformation of silicon. Optical scanning angles at resonant frequencies of 10.56 and 
$1.54 \mathrm{kHz}$ were obtained to be $27^{0}$ and $20^{0}$ for the two orthogonal axes, respectively, under driving voltages of $20 \mathrm{Vdc}$ plus $15 \mathrm{Vpp}$.

\subsubsection{In-plane comb drive arrangement}

In a recent research [8], three types of comb electrode structure are taken into consideration to find an optimal structure for driving the micro-mirror. Type I is a micromirror with the conventional rectangular comb-drive (Fig. 3 (a)). Type II is a micromirror with the slanted comb-drive (Fig. 3 (b)). The slanted comb-drive is generated from compromising the increase of rotation torque and the decrease of maximum static mechanical rotation angle due to placing comb fingers far from the rotation axis. This is due to the maximum static mechanical rotation angle is proportional directly to is the thickness of comb finger and inversely to the distance from the rotation axis of micro-mirror to the tip end of comb finger $L$. The geometrical parameters $t$ and $L$ are demonstrated in Fig. 1. Type III (Fig. 3 (c)) is a micro-mirror similar to type I, but additional combs are
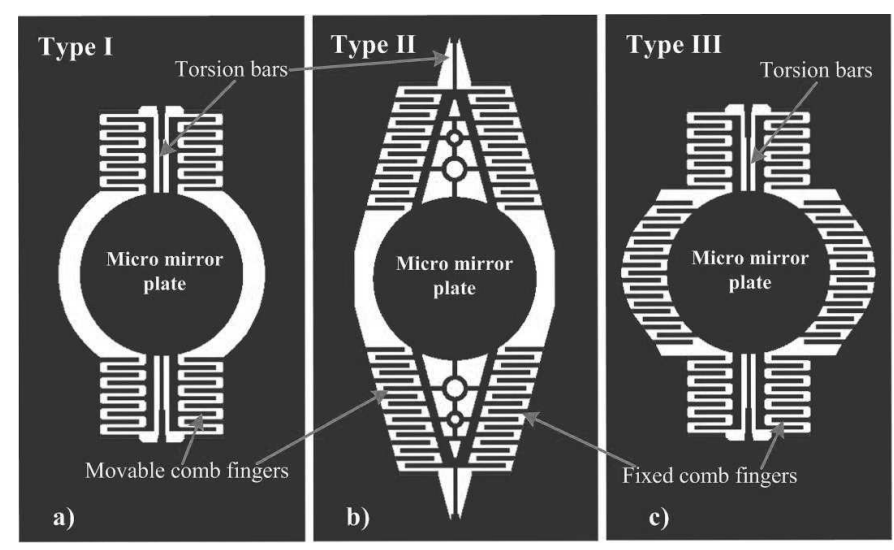

Fig. 3. Three different micro-mirror designs: a) micro-mirror with conventional comb (type I); b) micro-mirror with slanted comb (type II); c) micro-mirror with increased comb fingers on mirror plate (type III) [8]

Table 1. Characteristic values of mirrors calculated by the theoretical model [8]

\begin{tabular}{|c|c|c|c|c|}
\hline $\begin{array}{c}\text { Mirror } \\
\text { type }\end{array}$ & $\begin{array}{c}\text { Loss } \\
L_{\text {fric }}\left(10^{-10} \mathrm{~J}\right)\end{array}$ & $\begin{array}{c}\text { Stored Energy } \\
E_{\text {st }}\left(10^{-10} \mathrm{~J}\right)\end{array}$ & $\begin{array}{c}\text { Quality factor } \\
Q_{\text {fric }}\end{array}$ & $\begin{array}{c}\text { Static Torque } \\
T\left(10^{-9} N\right)\end{array}$ \\
\hline Type I & 3.19 & 68.9 & 135 & 1.41 \\
\hline Type II & 4.99 & 130 & 163 & 5.19 \\
\hline Type III & 8.01 & 64.8 & 50.8 & 7.62 \\
\hline
\end{tabular}

installed on the edges of mirror [5, 19]. However, the installation of combs on the mirror plane also increased the inertia and thus decreased the resonant frequency. Moreover, as seen in Tab. 1 although the electrostatic torque of type III is larger than 5.4 and 1.5 
times compared to that of type I and II, respectively, loss due to adding comb fingers on the mirror plane increases, which leads to the decrease of the quality factor. This is not desirable for the micro-mirror scanning under the resonant condition, which is due to the rotation angle at the resonant peak approximately estimated as a linear function of the quality factor. Since type II has the highest values of resonant frequency and quality factor, it is considered that type II has a better structure in the three types. To remove the air friction in the high velocity scanning inner mirror, the comb fingers are only placed on a low-velocity scanning outer drive frame [20]. The motion of the inner mirror is performed by a mechanical gain. However, this structure works only in coupling resonant mode, which is impossible for scanning control [21].

\subsection{Low voltage operation micro-mirror by decreasing air friction loss}

Under the resonant scanning, the mirror can be considered as a mechanical oscillator. The oscillation amplitude, which corresponds to the scan angle, is determined by

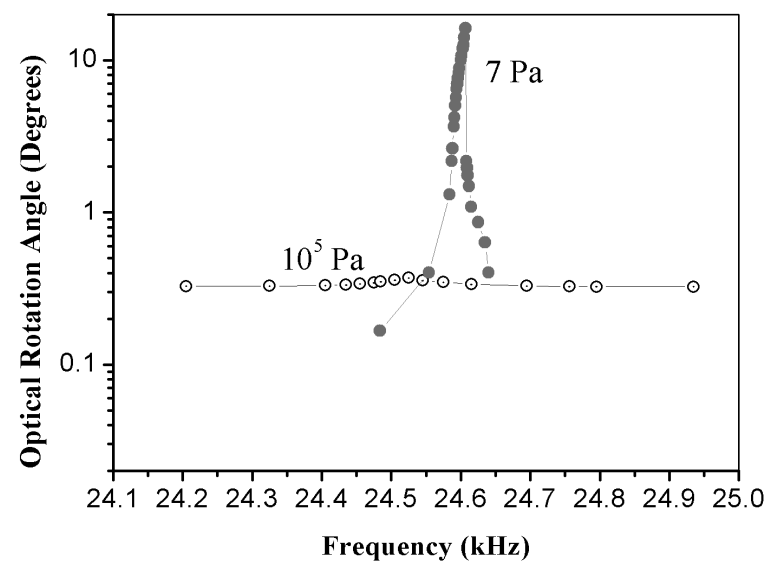

Fig. 4. The optical rotation angle of mirror type II (Fig. 3 (b)) measured as a function of frequency at the applied voltage of $60 \mathrm{~V}$ in atmospheric air and vacuum $(7 \mathrm{~Pa})[8]$

the balance between input energy and loss. If the loss of oscillating mirror is decreased, the scan angle of the mirror increases. It is considered that the air friction generates a large loss since the friction boundary of the comb-drive mirror is long. Therefore, it can be expected that the operation of micro-mirror in vacuum improves the scan angle and the driving voltage. Vacuum operation of vertical comb drive microdisplay-mirrors is systematically investigated both theory and experiment [8]. When pressure decreases from the atmospheric air to pressure lower than $1 \mathrm{~Pa}$, the quality factor $Q$ increases by about two orders of magnitude. As shown in Fig. 4, optical rotation angle of mirror type II (see Fig. 3 (b)) measured as a function of frequency at the atmospheric air and $7 \mathrm{~Pa}$ vacuum. The quality factor increases from 163 to 9735 when pressure is decreased from the atmospheric air to $7 \mathrm{~Pa}$ vacuum. The rotation angle increases 52 times larger than that in atmospheric air for micro-mirror type II, which corresponds to the decrease of operation voltage by a factor more than 7 . 


\subsection{Ultra-low voltage operation micro-mirror by decreasing air friction loss and anchor loss}

At low pressure, the quality factor of micro-mirror obtains a saturated value. This is due to energy loss causing by air friction completely removed $[8,10]$. Therefore, in order to decrease future the operation voltage of micro-mirror at resonant condition the other energy loss mechanisms need taken into consideration. Kotani et al. [22] proposed a structure of micro-mirrors connected in series as shown in Fig. 5, where a center mirror is connected to two side-mirrors by torsion hinges but the torsion hinges of center mirror are not connected to supporting points of substrate. The other sides of torsion hinges of the two side-mirrors are fixed to the supporting points of substrate. Since the motions of the series-mirrors are identical to a single mirror in a specific rotational mode, the loss by the supporting points of the center mirror is eliminated. The quality factor of the seriesmirrors is higher by about two orders than that of the single mirror (Fig. 6). Therefore,

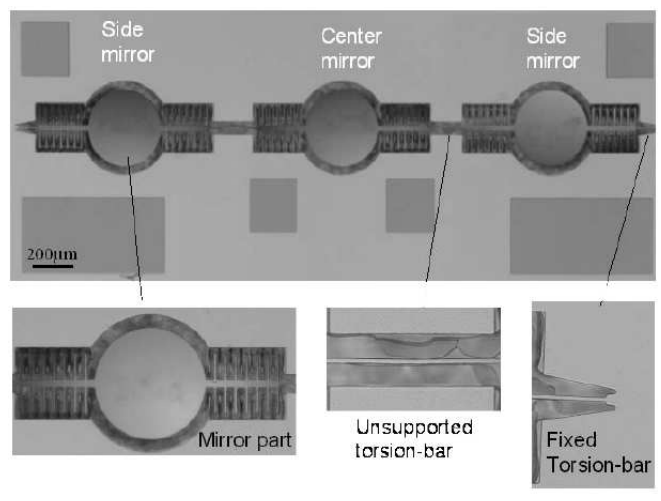

Fig. 5. Optical microscope images of the fabricated series micro-mirrors [22]

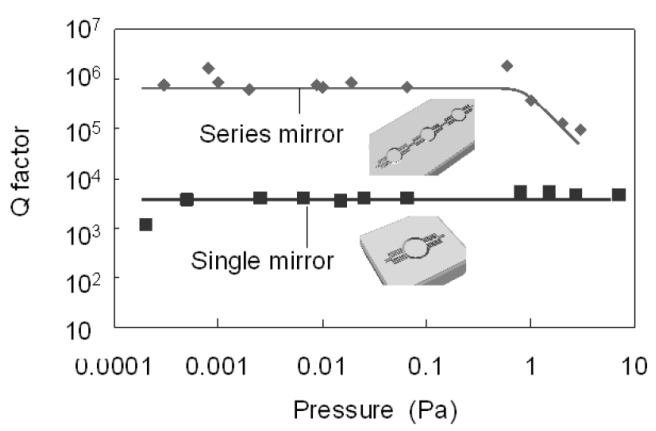

Fig. 6. $Q$ factors measured as a function of pressure for single and series-mirrors [22]

the operation voltage for the series-mirrors is smaller by a factor of two than that of the single mirror. 


\section{CONCLUSION}

In this paper, we reviewed recently proposed methods for decreasing the operation voltage of electrostatic comb drive micro-mirror. The comb actuator with narrow gap, vertically self-assembled combs, and angle vertical comb drive are considered to be effective for decreasing operation voltage. However, these methods are limited by fabrication process. Removing air friction by operating micro-mirror at low pressure is proved to be effective method for decreasing substantially operation voltage. An ultra-low micro-mirror can obtain by removing the air friction and anchor loss. Vacuum package is considered as a key technology to obtain a low voltage operation micro-mirror at high scan frequency and large rotation angle, which is an important step for the practice use of micro-mirror. Specially, the application of micro-mirror in laser scanning display is expected in near future.

\section{ACKNOWLEDGEMENT}

The devices were fabricated in Micro/Nano-Machining Research and Education Center, Tohoku University. This work is supported by JSPS.

\section{REFERENCES}

[1] Hane K. and Sasaki M., Micro-mirrors, Comprehensive Microsystems, 3 (2008), pp. 1-64.

[2] Sasaki M., Yuki S. and Hane K., Performance of tense thin-film torsion bar for large rotation and low-voltage driving of micromirror, IEEE journal of selected topics in quantum electronics, 13 (2007), 290-295.

[3] Fujita T., Maenaka K. and Takayama Y., Dual-axis MEMS mirror for large deflection-angle using SU-8 soft torsion beam, Sensors and Actuators A, 121 (2005), pp. 16-21.

[4] Sasaki M., Ishimori M., Song J. H. and Hane K., Tunable vertical comb for driving micromirror realized by bending device wafer, IEICE Trans Electron, E90-C (2007), 147-148.

[5] Ko Y.-C., Cho J.-W., Mun Y.-K., Jeong H.-G., Choi W. K., Kim J.-W., Park Y.-H., Yoo J.-B. and Lee J.-H., Eye-type scanning mirror with dual vertical combs for laser display, Sensors and Actuators A, 126 (2006), 218-226.

[6] Hirano T., Furuhata T., Gabriel K. J. and Fujita H., Design, fabrication, and operation of submicron gap comb-drive microactuators, J. Microelectromech. Syst., 1 (1992), 52-59.

[7] Ji C.-H., Choi M., Kim S.-C., Lee S.-H., Kim S.-H., Yee Y.and Bu J.-U., An electrostatic scanning micromirror with diaphragm mirror plate and diamond-shaped reinforcement frame, J. Micromech. Microeng., 16 (2006 ), 1033-1039.

[8] Manh C. H. and Hane K., Vacuum operation of comb-drive micro display-mirrors, J. Micromech. Microeng., 19 (2009) 105018 (8pp).

[9] Tachibana H., Kawano K., Ueda H., Noge H., Vacuum wafer level packaged two-dimensional optical scanner by anodic bonding, proc. MEMS 2009 (2009), 959-962.

[10] Chu H. M., Tokuda T., Kimata M., Hane K., Compact low-voltage operation micro-mirror based on high vacuum seal technology using metal can, J. Microelectromech. Syst., 19 (2010), 927-935.

[11] Bao M., Yang H., Squeeze film air damping in MEMS, Sensors and Actuators A, 136 (2007), $3-27$. 
[12] Pandey A. K. and Pratap R., A semi-analytical model for squeeze-film damping including rarefaction in a MEMS torsion mirror with complex geometry J. Micromech. Microeng., 18 (2008), 105003-14.

[13] Chung C.-K., Geometrical pattern effect on silicon deep etching by an inductively coupled plasma system, J. Micromech. Microeng., 14 (2004), 656-662.

[14] Conant R. A., Nee J. T., Lau K. Y., and Muller R. S., A flat high-frequency scanning micromirror, Solid-State Sensor and Actuator Workshop Technical Digest, (2000), pp. 6-9.

[15] Milanovic' V., Kwon S., and Lee L. P., High Aspect Ratio Micromirrors With Large Static Rotation and Piston Actuation, IEEE Photonics Technology Letters, 16 (2004), 1891-1893.

[16] Hah D., Patterson P. R., Nguyen H. D., Toshiyoshi H., and Wu M. C., Theory and experiments of angular vertical comb-drive actuators for scanning micromirrors, IEEE J. Sel. Top. Quantum Electron., 10 (2004), pp. 505-13.

[17] Kim J., Choo H., Lin L., and Muller R. S., Microfabricated Torsional Actuators Using SelfAligned Plastic Deformation of Silicon, J. Microelectromech. Syst. 15, (2006), 553-561.

[18] Kim J., Christensen D., and Lin L., Monolithic 2-D scanning mirror using self-aligned angular vertical comb drives, IEEE Photonics Technology Letters, 17 (2005), 2307-2309.

[19] Wu M., Lin H.-Y. and Fang W., Design of novel sequential engagement vertical comb electrodes for analog micromirror, IEEE Photonics Technology Letters, 19 (2007), 1586-1588.

[20] Arslan A., Brown D., Davis W., Holmstrom S., Gokce S. K., Urey H., Comb Actuated Resonant Torsional Microscanner with Mechanical Amplification, IEEE J. Microelectromechanical Systems, 19 (2010), pp. 936-943.

[21] Tsai J. C., Lu L. C., Hsu W. C., Sun C. W. and Wu M. C, Linearization of a two-axis MEMS scanner driven by vertical comb-drive actuators, J. Micromech. Microeng., 18 (2008), 015015 (8pp).

[22] Kotani S., Chu H.M., Sasaki T., and Hane K., Micro-mirrors connected in series for lossmechanism study and low-voltage operation, Transducers'11 (2011), pp. $554-557$.

Received July 31, 2012 